\title{
ESTUDO DA ESTABILIDADE ATMOSFÉRICA EM PARQUES EÓLICOS
}

Yoshiaki Sakagami, Pedro Alvim de Azevedo Santos, Reinaldo Haas, Júlio César Passos, Frederico de Freitas Taves

Universidade Federal de Santa Catarina/Dep. Eng. Mecânica/LEPTEN

\section{RESUMO}

Este trabalho apresenta os resultados preliminares sobre a estabilidade atmosférica em dois parques eólicos em operação no nordeste brasileiro, utilizando cinco técnicas de cálculo. Uma comparação entre as diferentes técnicas mostram uma coerência entre as técnicas de eddy correlation, gradiente e coeficiente de cisalhamento nos dois parques. Em Pedra do Sal-PI estas três técnicas consideram a atmosfera no local, predominantemente convectiva, e em Beberibe-CE, entre neutra e convectiva. Por outro lado, as técnicas baseadas na intensidade de turbulência e na energia cinética turbulenta divergem dos demais e classificam a atmosfera como muito estável em Pedra do Sal.

Palavras-chave: estabilidade atmosférica, eddy covariance, energia eólica, parques eólicos

\section{INTRODUÇÃO}

Recentemente, os novos aerogeradores têm alcançado $127 \mathrm{~m}$ de diâmetro de rotor e, consequentemente, tem ocorrido uma necessidade de se avaliar o potencial eólico através do perfil de velocidade do vento ao longo do rotor. Entretanto, este perfil pode ser alterado em função da estabilidade da atmosfera, e assim, influenciar no desempenho da produção de energia elétrica em parques eólicos (WHARTON; LUNDQUIST, 2010). O presente trabalho tem o objetivo avaliar a estabilidade atmosférica em dois parques eólicos em operação no nordeste brasileiro, comparando os resultados de cinco diferentes técnicas, utilizando dados de torres anemométricas de $100 \mathrm{~m}$ de altura.

\section{MATERIAIS E MÉTODOS}

Os parques eólicos estudados estão localizados nas cidades de Beberibe/CE e Pedra do Sal/PI. Cada parque está equipado com uma torre anemométrica de $100 \mathrm{~m}$ de altura. O 
presente estudo utilizou dados do anemômetro sônico 3D RM-YOUNG 8100, instalado a $100 \mathrm{~m}$, bem como os dados de temperatura (a $100 \mathrm{~m}$ e $40 \mathrm{~m}$ ) e velocidade (a $100 \mathrm{~m}, 60 \mathrm{~m}$ e 40m). Os resultados foram calculados com dados referentes ao mês de Julho de 2013. Os dados do anemomêtro sônico 3D são coletados com uma taxa de aquisição de $20 \mathrm{~Hz}$, com médias a cada 10min. Foram utilizados cinco técnicas para classificação da estabilidade atmosférica: eddy correlation (Eddy), gradiente, coeficiente de cisalhamento $(\alpha)$, energia cinética turbulenta (ECT) e intensidade de turbulência (IT). O método de eddy correlation realiza o cálculo do comprimento de Monin-Obukov (L) a partir da estimativa dos fluxos turbulentos de calor e de quantidade de movimento (Eq. 1). Arya (2001) apresenta o método do gradiente, a partir do cálculo de gradientes de temperatura e de velocidade em uma altura média, $\mathrm{Z}_{\mathrm{m}}$, do número de Richardson, Ri, e, consequentemente, do comprimento L (Eq. 2).

$$
L=-\frac{u_{*}{ }^{3}}{\left[\kappa\left(\frac{g}{T_{0}}\right)\left(\frac{H}{\rho c_{p}}\right)\right]} \quad \text { (1) } \quad L=\frac{z_{m}}{R i}=z_{m}\left[\frac{\frac{g}{T_{0}} \frac{\partial \theta}{\partial z}}{\left(\frac{\partial U}{\partial z}\right)^{2}}\right]^{-1}
$$

Wharton e Lundquist (2010) realizaram uma classificação de estabilidade a partir de medições da energia cinética turbulenta (Eq. 3), ao definir faixas de ocorrência de acordo com o parâmetro adimensional de Monin-Obukov, z/L.

$$
E C T=\frac{1}{2}\left(\overline{u^{\prime} u^{\prime}}+\overline{v^{\prime} v^{\prime}}+\overline{w^{\prime} w^{\prime}}\right)
$$

O mesmo procedimento da ECT foi aplicado ao parâmetro intensidade de turbulência (Eq.4). A técnica do coeficiente de cisalhamento ( $\alpha$ ), busca ajustar um perfil de velocidades a cada classe de estabilidade atmosférica, através do perfil de lei de potência (Eq. 5).

$$
I T=\sigma_{U} / \bar{U} \quad(4) \quad U(z)=U_{r}\left(\frac{z}{z_{r}}\right)^{\alpha}
$$

A estabilidade atmosférica foi dividida e avaliada em classes conforme Wharton e Lundquist (2010) (Tab. 1). 
Tabela 1 - Classificação para a estabilidade atmosférica.

\begin{tabular}{|c|c|c|c|c|}
\hline Classe & $\mathbf{L}$ & ECT & IT & Alfa \\
\hline Muito Estável & $0<\mathrm{L}<50$ & ECT $<0,4$ & IT $<8 \%$ & $\alpha>0,3$ \\
\hline Estável & $50<\mathrm{L}<200$ & $0,4<$ ECT $<0,6$ & $8 \%<$ IT $<10 \%$ & $0,2<\alpha<0,3$ \\
\hline Neutra & $200<\mathrm{L}<-300$ & $0,6<\mathrm{ECT}<1,0$ & $10 \%<\mathrm{IT}<20 \%$ & $0,1<\alpha<0,2$ \\
\hline Convectiva & $-300<\mathrm{L}<-15$ & $1,0<\mathrm{ECT}<1,4$ & $20 \%<\mathrm{IT}<30 \%$ & $0,0<\alpha<0,1$ \\
\hline Muito convectiva & $-15<\mathrm{L}<0$ & $\mathrm{ECT}>1,4$ & IT $>30 \%$ & $\alpha<0,0$ \\
\hline
\end{tabular}

Fonte: Wharton e Lundquist (2010).

\section{RESULTADOS E DISCUSSÕES}

Os resultados para Pedra do Sal (Fig. 1-a) apresentam uma coerência entre as técnicas de Eddy, gradiente e coeficiente alfa sendo o primeiro considerado o mais confiável para avaliação dos fluxos (ARYA, 2001).

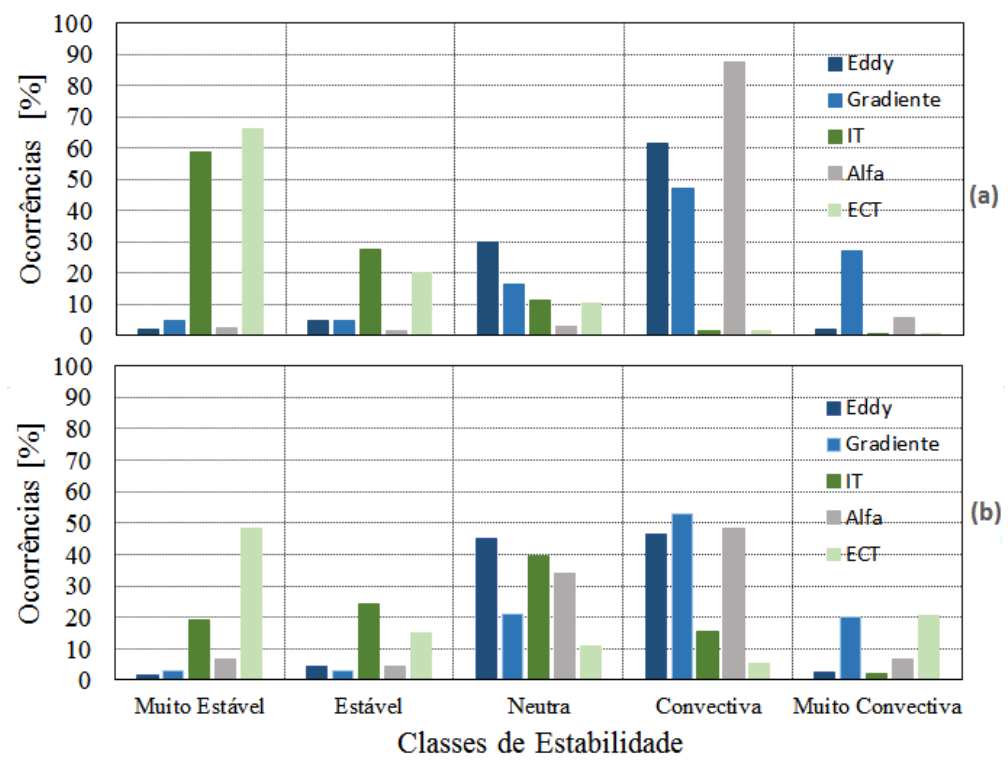

Figura 1 - Resultados para estabilidade em Pedra do Sal (a) e Beberibe (b).

As três técnicas utilizadas indicam uma atmosfera predominantemente convectiva com 61,4\% (Eddy), 46,9\% (gradiente) e 87,3\% ( $\alpha$ ) de ocorrências neste período. Este resultado está de acordo com os resultados apresentados por Peña, Gryning e Hasager (2008), por tratar-se de uma condição marítima. As técnicas de ECT e IT tendem para uma atmosfera muito estável com $66,3 \%$ e 58,9\% de ocorrências, respectivamente. Para os 
resultados de Beberibe (Fig. 1-b), a atmosfera é classificada entre neutra e convectiva utilizando as técnicas de Eddy, gradiente e coeficiente alfa. A técnica de ECT aponta para uma atmosfera muito estável $(48,5 \%)$ e a IT mostra-se distribuída entre as classes, exceto para a classe muito convectiva, indicando maior ocorrência para uma atmosfera neutra $(39,4 \%)$.

\section{CONCLUSÕES}

Os resultados preliminares sobre a estabilidade atmosférica nos parques eólicos de Pedra do Sal e Beberibe mostram-se satisfatórios e de acordo com outros resultados encontrados na literatura que também utilizam as técnicas de Eddy, gradiente e coeficiente alfa. As técnicas de ECT e IT não apresentaram resultados conforme o esperado o que sugere uma melhor análise e ajuste quanto aos limites de cada classe de estabilidade. Esses resultados podem contribuir para uma melhor estimativa da produção de energia elétrica dos aerogerados através do cálculo do perfil de velocidade do vento ao longo do seu rotor, com suas devidas correções conforme a estabilidade atmosférica.

\section{AGRADECIMENTOS}

Os autores agradecem o apoio do CNPq, ANEEL e Tractebel Energia.

\section{REFERÊNCIAS}

[1] ARYA, S. P. Introduction to Micrometeorology. $2^{\circ}$ Ed. London: Academic Press, 2001.

[2] PEÑA, A.; GRYNING, S. E.; HASAGER, C. B. Measurements and Modelling of the Wind Speed Profile in the Marine Atmospheric Boundary Layer. Boundary-Layer Meteorology, v.129, p. 479-495, Oct. 2008.

[3] Wharton, S.; LUndQUist, J. K. Atmospheric Stability Impacts on Power Curves of Tall Wind Turbines - An analysis of a west coast North American wind farm. Lawrence Livermore National Lab, Livermore, 2010. 